\title{
Post Traumatic Stress Disorder: Current Issues and Future Trends
}

\section{Amit Nandan Dhar Dwivedi*}

Department of Radiodiagnosis \& Imaging, Institute of Medical Sciences, Banaras Hindu University, India

Although hints of such diagnosis are present in countless experiences of world wars and innumerous world literature, Post traumatic stress disorder (PTSD) was first described in 1952 by Diagnostic and Statistical Manual of Mental Disorders (DSM)-I by the nomenclature "gross stress reaction". DSM-III in the mid-1980s thoroughly examined the disorder and reintroduced it as posttraumatic stress disorder (PTSD) in background of resurged scientific interest provoked by the recent occurrence of the Vietnam War. PTSD is distinctive among psychiatric conditions because of the extraordinary emphasis placed upon the traumatic stresssor as causative factor. The traumatic stressor can be any bitter event outside the usual human experiences such as wars, rape, torture, accidents and other mass catastrophes. Nonetheless, the degree of alteration depends on the individual vulnerability and resilience. The psychiatric manifestation of the disease is characterised by intrusive recollections, avoidance, negative cognition and altered reactivity. In present day scenario, the existence of a distinctive and valid psychiatric entity is unquestioned; however there are yet ambiguities and gray areas to be resolved especially with the re-emergence of biological aspect of this entity and treatment options on the horizon.

We congratulate and thank the authors of this special issue who have beautifully dealt with various aspects of this disorder from the cross cultural and epidemiological perspective, the exiting and evolving treatment protocols and assessment of associated comorbidities. We also take this opportunity to thank the reviewers for their valuable contribution to ensure high standards of the papers. The topics explored in this special issue include: "Acute Post-Traumatic Stress Reactions in Children Survivors of a Large Road Traffic Accident: Epidemiological Analysis and Eye Movement Desensitization and Reprocessing Treatment", "Association between trauma history and juvenile sexual offending", "Eye Movement Desensitisation and Reprocessing Treatment of Posttraumatic Stress Disorder, Comorbid Disorders and Personality Traits: A Case Series with 12 Month Follow Up" and "Treatment, outcome After Traumatic Brain Injury, Impacts on LongTerm Outcome after Major Trauma-Traumatic Brain and Orthopedic Injuries". The topic "Psychological Trauma: Experience from Iraq Hypnosis for PTSD: Evidence Based Placebo-Controlled Studies and Current Opinions on Epidemiology" analyses the status of this disease in Iraq which has been battleground for successive conflict since years and supposedly harbour this disorder with increased prevalence. Furthermore innovative topics related to Trauma such as Cellular Response of the Tendon Sheath in Tendon Injury: Experimental Research in New Zealand Rabbits and Surgical Outcomes of Pediatric Humeral Supracondylar Fractures Treated By Posterior Approach has been discussed. It is hoped that this issue will make a good reference material and be of great use for the concerned physicians.
*Corresponding author: Amit Nandan Dhar Dwivedi, Department of Radiodiagnosis \& Imaging, Institute of Medical Sciences, Banaras Hindu University, India, Tel: +91-9793856930; E-mail: amitnandan21@yahoo.com

Received January 15, 2014; Accepted January 19, 2014; Published January 21, 2014

Citation: Dhar Dwivedi AN (2014) Post Traumatic Stress Disorder: Current Issues and Future Trends. J Trauma Treat 3: e117. doi:10.4172/2167-1222.1000e117

Copyright: (c) 2014 Dhar Dwivedi AN. This is an open-access article distributed under the terms of the Creative Commons Attribution License, which permits unrestricted use, distribution, and reproduction in any medium, provided the original author and source are credited. 